\title{
Publisher's Note: Sliced Basis Density Matrix Renormalization Group for Electronic Structure [Phys. Rev. Lett. 119, 046401 (2017)]
}

E. Miles Stoudenmireø and Steven R. White

(Q) (Received 18 March 2021; published 9 April 2021)

DOI: 10.1103/PhysRevLett.126.149901

This Letter was published online on 24 July 2017 with an error in an Award number in the last paragraph of the article. The last paragraph of the article should read "We acknowledge support from the Simons Foundation through the Many-Electron Collaboration and from the U.S. Department of Energy, Office of Science, Basic Energy Sciences under Award No. DE-SC0008696." The Award number has been corrected as of 24 March 2021. The Award number is incorrect in the printed version of the journal. 